\title{
Comparative transcriptome analysis reveals storage carbohydrates related genes in kelp Saccharina japonica
}

\section{Linan Zhang}

Qingdao Agricultural University

\section{Zengmei Cao}

Shandong Oriental Ocean Sci-tech Co., Ltd

\section{Guangjin Liang}

Shandong Oriental Ocean Sci-tech Co., Ltd.

\section{Xiaojie Li}

Shandong Oriental Ocean Sci-tech Co., Ltd

Hao Wu ( $\nabla$ wuhaoqau@163.com )

Qingdao Agricultural University https://orcid.org/0000-0002-4503-7128

\section{Research article}

Keywords: Saccharina japonica, sporophyte, transcriptome, carbohydrate, alginate, mannitol

Posted Date: May 14th, 2019

DOl: https://doi.org/10.21203/rs.2.9606/v1

License: (9) This work is licensed under a Creative Commons Attribution 4.0 International License. Read Full License 


\section{Abstract}

Background Saccharina japonica is a brown macroalga commercially cultivated in China for almost a century. As a natural raw material, it has been widely used to extract carbohydrates, e.g. alginate and mannitol, for food, pharmaceutical, and potentially for biofuel production. However, the molecular mechanisms of biosynthesis and regulation of these compounds in this species are not comprehensive and less well understood. Results In this study, we performed whole transcriptome and differential transcript expression analyses of S. japonica sporophyte of distinct developmental stages. Combined with the phenotype of blade and the content of alginate and mannitol, the sporophyte could be divided into four developmental stages. Accordingly, 589 million clean reads were generated, and 98,497 novel transcripts and 4,514 novel genes were identified by transcriptome analysis. Gene expression analysis revealed that 2,542 genes were differentially expressed. KEGG analysis indicated that these genes were significantly enriched in "Carbon metabolism", "Photosynthesis", and "Photosynthesis-antenna proteins" pathways, which were important for carbohydrates metabolism during sporophyte development. Through a systematic analysis, the genes encoding enzymes for biosynthesis of cell wall carbohydrates (including alginate, fucoidan and cellulose) and cytoplasm storage carbohydrates (mannitol, laminarin and trehalose) were identified, representing good candidates for further functional studies. Conclusion The work presented is the first transcriptome analysis of sporophytes development and provides a dynamic view of carbohydrate metabolism process and an important resource for functional genomics studies in $\mathrm{S}$. japonica. The results obtained lay the basis for elucidating the molecular mechanism of carbohydrate biosynthesis and for genetic breeding of carbohydrates-related traits in kelp.

\section{Background}

Brown algae are the only photoautotrophic and multicellular organisms in the Stramenopile, which evolved independently from Opisthokonts (including animals and fungi) and Archaeplastida (including terrestrial plants, green and red algae) [1]. Brown algae contain complex and diverse carbohydrate components, which can be divided into two categories. One group of carbohydrates is involved in the main components of the cell wall, including alginate, fucoidan and cellulose [2]. The other group of carbohydrates is synthesized and stored in cytoplasm, including mannitol, laminarin and trehalose [3]. Among them, alginate and mannitol are unique carbohydrates in brown algae, which are different from those in plants, animals, fungi, and even in other Stramenopiles, including diatoms and oomycetes. Nowadays, these storage carbon compounds have been widely used in food, pharmaceutical, industrial production [4-7]. In addition, alginate and mannitol also showed great potential for biofuel production with the establishment of high-efficiency ethanol production recently [8-10]. Kelp (Saccharina japoinca) is a major, commercially cultivated brown alga in China. Due to the high biomass productivity, it has been widely used for commercially available carbohydrates (including alginate and mannitol) extraction as the main natural raw material. In this perspective, thorough understanding the molecular mechanisms of biosynthesis and regulation of these carbohydrates compound in this species provides the prerequisite 
for further utilization of these polysaccharides in and designing of optimized target biomass characteristics in breeding.

Recently, based on the complete genome sequences and annotated information of a model brown alga Ectocarpus sp. [11], the great progress has been made in the biosynthesis pathways of brown algal carbohydrates, including the identification of the candidate key genes, phylogenetic analyses of the key enzymes and reconstruction of the routes for carbohydrates biosynthesis [12-17]. Taking alginate as an example, six of the 13 proteins involved in alginate biosynthesis in bacteria were identified in Ectocarpus, including GDP-mannose-6-dehydrogenase (GMD) and mannuronate C5-epimerase (MC5E), which catalyzed the rate-limiting step from GDP-mannose into GDP-mannuronic acid and the terminal step from D-mannuronate into L-guluronate, respectively [15]. Taking mannitol as an example, a complete set of enzymes for mannitol synthesis was found in Ectocarpus [14]. Evolutionary analysis showed that the biogenesis of both alginate and mannitol in brown algae may be acquired by horizontal gene transfer (HGT) from Actinobacteria, while the metabolism of laminarin and fucoidan in brown algae is probably an ancestral pathway in Stramenopiles or even in other Eukaryotic phyla. These results provide new insights into the origin and evolution of eukaryotic polysaccharides, and facilitate to deeply understand the molecular regulated mechanism of polysaccharides metabolism in brown algae. However, the lift cycle of Ectocarpus involves an alternation between the diploid sporophyte and haploid gametophyte. Both sporophyte and gametophyte are single-row cell filaments with similar phenotypic characteristics. The significant dynamic changes in content of various carbohydrates were reported neither in sporophyte development nor in gametophyte development so far. This limited information restricts the understanding of the carbohydrates biosynthesis as well as its correlation with content change in brown algae.

In contrast to Ectocarpus, the S. japonica sporophyte, which is dominant in its life cycle, is macroscopic and thalloid. They have been widely used to extract alginate, mannitol and laminarin for various applications in China. Many studies have shown that the content of carbohydrates exhibits a significant change during the sporophyte development in Laminariales [18-21]. There are also evidences for seasonal fluctuations of alginate content and mannitol content in $S$. japonica sporophyte [22]. Thus, $S$. japonica sporophytes may be an ideal material for systematic analysis of carbohydrates metabolic pathways in brown algae, and it can be assumed that the genes involved in carbohydrates metabolism could be efficiently identified on the basis of reasonable sampling. Although the whole genome of $S$. japonica has been sequenced [23], and some candidate genes involved in the biosynthesis of carbohydrates, including alginate, fucoidan, mannitol, and laminarin, have been identified [24-28], the molecular mechanisms of biosynthesis and regulation of these compounds in this species are not comprehensive and less well understood.

In this study, transcriptome datasets of blades tissues of four different sporophyte developmental stages have been established by RNA-seq technology, according to the phenotypic characteristics and the content changes of alginate and mannitol of cultured $S$. japonica. Through the comparative transcriptome analysis, the expression profile of differentially expressed genes (DEGs) was categorized and characterized, and the candidate genes underlying storage carbohydrates metabolism were 
identified. The results will deepen our understanding of underlying molecular process of carbohydrates biosynthesis and facilitate the breeding of carbohydrates-related traits in $S$. japonica.

\section{Results}

Changes of alginate and mannitol content during sporophytes development

To investigate the formation and regulation of alginate and mannitol, the dynamic changes of alginate content and mannitol content were studied during sporophytes development (Figure 1). The content of alginate gradually increased since SJsp1 (January), reaching the highest level at SJsp3 (March). After a gradually declined in SJsp4 (Apirl) and SJsp5 (May), the alginate content increased significantly again in SJsp6 (July) (Figure 1a). The mannitol content was lowest in SJsp1, and gradually increased in SJsp2 and SJsp3. Its content remained at a high level in the later stages of development although there appeared some fluctuations in SJsp4 and SJsp5 (Figure 1b). These results suggested that alginate and mannitol were differentially controlled at distinct developmental stages.

Analysis of RNA-seq libraries

According to the above biochemical analysis, samples from four major development stages (SJsp1, SJsp3, SJsp5 and SJsp6, respectively) were used for RNA-seq. In total, twelve cDNA libraries were constructed, sequenced and generated 634.9 million sequence reads totally in this study. After removing of low-quality reads and adaptor sequences, 589 million clean reads were kept and then mapped to the reference genome of $S$. japonica using HISAT [29]. Furthermore, out of high-quality reads generated from the twelve samples, uniquely mapped reads were 68.55 to $75.46 \%$, while total mapped reads were 83.92 to $90.29 \%$ (Table 1). A total of 98,497 novel transcripts were identified in which 27,079 of them are previously unknown splicing event for known genes and 4,514 of them are novel coding transcripts without any known features (Table 2 and Additional file 1: Table S1).Thus, 21,225 genes were identified in this study, including 16,793 known genes and 4,514 novel genes.

\section{Analysis of DEGs during sporophyte development}

To identify significant DEGs during $S$. japonica sporophytes development, the expression quantity of each gene in four libraries was compared pairwise and filtered. A total of 2,542 DEGs were detected among four libraries, accounting for $11.98 \%$ of total unigenes (Additional file 2: Table S2). To provide a global view of gene expression, a heatmap was generated using normalized FPKM value. Hierarchical clustering revealed that the expression could be classified into several distinct patterns (Figure 2). The different expression patterns among the four stages revealed that the maximum differences (610 up-regulated genes and 832 down-regulated genes) were examined between the SJsp1 and SJsp 5 stages, followed by SJsp1 and SJsp6 stages (716 up-regulated genes and 569 down-regulated genes). The minimum differences (132 up-regulated genes and 53 down-regulated genes) were examined between the SJsp5 and SJsp6 stages (Figure 3). 
To obtain a detailed perspective on the functions of DEGs, GO and KEGG enrichment analysis were conducted. A total of 3,712 GO terms including 1,494 (40.25\%) cellular component terms (CC), 1,324 (35.67\%) biological process terms (BP), and 894 (24.08\%) molecular function terms (MF) were assigned to 2,542 DEGs (Table 3). Analysis of level $2 \mathrm{GO}$ term distribution showed that in all comparisons "metabolic process" and "cell process", "cell" and "cell part", "catalytic activity" and "binding" were the most common annotated terms in the BP, CC and MF, respectively (Additional file 6: Figure S1).

The DEGs were allocated to 116 pathways. Based on KEGG pathway enrichment analysis, the pathway "Photosynthesis-antenna proteins" was significantly enriched in SJsp1 vs SJsp5, SJsp1 vs SJsp6, SJsp3 vsSJsp 5 and SJsp3 vs SJsp6. The pathway "Photosynthesis" was also significantly enriched in SJsp1 vs SJsp6 and SJsp3 vs SJsp6. Genes associated with "Carbon metabolism" pathway were significantly enriched in SJsp3 vs SJsp6. In addition, lipid metabolism represented the dominant terms. Genes involved in "Cutin, suberine and wax biosynthesis" and "Arachidonic acid metabolism" pathways were significantly enriched in SJsp3 vs SJsp5 and SJsp5 vs SJsp6, respectively. The pathway "Linoleic acid metabolism" was significantly enriched in SJsp1 vs SJsp3 and SJsp1 vs SJsp6 (Additional file 3: Table s3).

\section{Genes involved in alginate biosynthesis}

Given that alginate is the most abundant polysaccharide component in the cell wall of brown algae, the genes related to alginate pathway were first investigated. Thirty-one alginate genes involved in four catalyzed reactions were identified in this study, including three mannose-6-phosphate isomeras (MPI) genes, two phosphomannomutase (PMM) genes, four GDP-mannose 6-dehydrogenase (GMD) genes and 22 mannuronate $\mathrm{C} 5$-epimerase (MC5E) genes. The expression levels of these genes at four different developmental stages were showed in Figure 4. GMD catalyses a rate-limiting step of the alginate pathway, which converts the GPD-mannose into GDP-mannuronic acid. Phylogenetic analysis showed that four GMDs of $S$. japonica cluster in a distinct clade with that of other brown algae species, including Ectocarpus and Sargassum binderi (Additional file 6: Figure S2). Three GMD genes (SjGMD2-SJ11025, SjGMD3-SJ11033 and SjGMD4-SJ11024) were identified to be differentially expressed during sporophytes development (Figure 4). Among them, the expression levels of SjGMD3-SJ11033 and SjGMD-SJ11025 remained at high level and its expression profiles exhibited a similar pattern with the content of alginate (Figure 1 and Figure 4), possibly reflecting its key role in alginate biosynthesis.

GDP-mannose was converted from fructose-6-phosphate by three upstream genes, MPIs, PMMs, and MPGs. The two MPIgenes, SjMPI1-Sj22044 and SjMPI4-SJ15067, were also showed a similar expression pattern with the content of alginate, but only the SjMPI4-SJ15067 was expressed differently during sporophyte development (Figure 4 and Figure 6). The MPG gene was not found in this study, which convert mannose-1-phosphate into GDP-mannose. Interestingly, it has been reported that SjMPI4 encodes a functional MPG protein, suggesting that SjMPI4-SJ15067 encoding protein may be a bifunctional enzyme in alginate pathway, as the role of AlgA in bacteria [25, 30]. There is one PMMgene (SjPMM- 
SJ14545) and one PMM/phosphoglucomutase (PGM)gene (SjPMM/PGM-SJ02279) in S. japonica transcriptome. The phylogenetic tree of PMM revealed that SjPMM/PGM belonged to a single group which including PMM/PGMs of other eukaryotic diatom and prokaryotic bacteria and SjPMM formed another group, indicating that they are evolved independently (Additional file6: Figure S3). It has been reported that SjPMM/SjPGM was closely related to alginate synthesis but exhibits a remarkable preference for Glucose-1-P over Mannose-1-P [28]. Expression analysis showed that the expression level of SjPMM-SJ14545 was always higher than SjPMM/PGM-SJ02279 during developmental stages, indicating that SjPMM-SJ14545 might play a major role in the reversibly catalytic reaction between M1P and Mannose-6-P (Figure 4). Following the formation of the alginate precursor GDP-mannuronic acid by above biochemical reactions, the alginate biosynthesis involves polymerization and epimerization by mannuronan synthase (MS) and MC5E, respectively. However, no MS genes were identified although two GTs family genes may be candidates for catalyzing the polymerization of activated mannuronate units in Ectorcarpus. A total of 77 unigenes were annotated as members of MC5E genes, and then 21 complete and one partial MC5Es genes were found through the local BLASTX search using characterized MC5E genes in brown algae. The phylogenetic tree showed that SjMC5Es were divided into three clades, of which most of SjMC5Es and six reported MC5Es of Laminaria digitata belonged to the one clade. Among them, three MC5Es of L. digitata (LdMC5E-1, LdMC5E-2, and LdMC5E-4) and three SjMC5Es (SjMC5ESJ22025, SjMC5E-SJ22027, and SjMC5E-SJ22031) clustered into a subclade. Three MC5Es of L. digitata (LdMC5E-3, LdMC5E-5, and LdMC5E-6) first clustered with its orthologs from S. japonica (SjMC5ESJ07285, SjMC5E-SJ07251 and SjMC5E-SJ07248, respectively) then formed a sister clade with other three SjMC5Es (Additional file 6: Figure S4). Further expression analysis showed that all the orthologous genes of LdMC5Es in S. japonica were identified to be differentially expressed during sporophyte development except SjMC5E-SJ07285 (Figure 4). In addition, the distinct the expression patterns were detected in these differentially expressed SjMC5E genes. Four genes (SJ22025, SJ22027, SJ22031, SJ07248) showed preferential expression in SJsp1, whereas one gene (SJ07251) was expressed at higher levels in SJsp6, indicated their different roles in alginate modification. These results suggested that the catalytic reaction involved MC5E is likely more complicated in alginate biosynthesis pathway and indicated putative roles of these SjMC5E genes in biosynthesis of different kinds of alginate in kelp.

\section{Analysis of putative genes related to fucoidan and cellulose biosynthesis}

Fucoidan and cellulose are the main cell wall components of the brown algae, therefore their biosynthesis pathway were further investigated. The fucoidan biosynthesis route shared the same first three steps with alginate pathway, and involved GDP-mannose 4, 6-dehydratase (GM46D), GDP-fucosesynthetase (GFS), fucosyltransferase (FS), and sulfotransferase (ST) in the subsequent reaction steps (Additional file 6: Figure S5). Two SjGM46D genes (SJ21655 and SJ04008) and one SjGFS gene (SJ05880) could be involved in converting GDP-mannose to GDP-fucose, and then $11 \mathrm{SjFS}$ genes might be associated with the polymerization of GDP-fucose into the elongating fucan chain. In addition, 26 members of ST genes were found in S. japonica transcriptome. Among them, six SjST genes (BGI_novel_G002583, BGI_novel_G002584, BGI_novel_G002585, SJ08073, SJ0108, and SJ21424) are homologous to those EsST genes, which are candidates to function as fucan STs, suggesting that these genes might be 
responsible for the biosynthesis of fucoidan rather than other various secondary metabolites. One fucokinase gene (SjFK-SJ13948) exists in S. japonica, which encoded 1,633 amino acid residues with putative ORF of 4,902 bp and contained two fucokinase superfamily domains. A comparison of SjFK with EsFK showed that SjFK domain-1 shared 72.73\% identify with that of EsFK (CBJ29017) and SjFK domain-2 shared $72.39 \%$ identify with that of EsFK (CBJ29015), suggesting that SjFK gene might encode a bifunction enzyme with both fucokinase and GDP-fucose pyrophosphorylas (GFPP) activity. These results further provided evidence for the existence of an alternative salvage pathway for fucoidan biosynthesis in brown algae, in which GDP-fucose can be yield from L-fucose.

Cellulose synthase (CESA) and cellulose synthase-like (CSL) genes are members of the GT2 family, which are mainly involved in the biosynthesis of cellulose and hemicelluloses components in plant. There are 8 complete CESAs were identified and clustered into two distinct clades. Four proteins homologous to EsCESAs were belonged to CESAs subclade, while other proteins homologous to EsCSLs clustered into CSL subclade (Additional file 6: Figure S6a). Among them, most genes can only be detected with very low expression levels during the sporophyte development. In contrast, the expressions of one SjCESA gene (SJ01458) and one SjCSL gene (SJ21252) were relatively lower in the stage of SJsp1, but was significantly increased in the subsequent developmental stages, including SJsp3, SJsp5 and SJsp6 (Additional file 6: Figure S6b). The expression patterns of these two genes were consistent with the physiological process of kelp that the synthesis of a large number of cell walls was required, indicating putative roles of them in the biosynthesis of cellulose and hemicelluloses, respectively.

Genes involved in mannitol biosynthesis

In brown algae, mannitol biosynthesis from the photoassimilate D-fructose-6-phosphate is catalyzed by mannitol-1-phosphate-5-dehydrogenase (M1PDH) and mannitol-1-phosphatase (M1Pase), and its degradation involves mannitol-2-dehydrogenase (M2DH) and Fructokinase (FrK). Two SjM1PDH genes and two SjM1Pase genes were found in this study, respectively (Figure 5). M1PDHs from two brown algae species (S. japonica and E. siliculosus) and two Prasinophytes species belong to one clade, while those from fungi and Actinobacteria cluster into another clade, suggesting the independent evolution of M1PDHs in each branch (Additional file 6: Figure S7). The congruence of the phylogenetic tree was obtained for same evolutionary relationship was also showed in phylogenetic tree of M1Pases (Additional file 6: Figure S8). Interestingly, an example of functional clustering, which has been reported in Ectocarpus, was also found in S. japonica. The SjM1DPH gene (SJ20113) and the SjM1Pase gene (SJ20112) are adjacent on the genome, as the M1DPH gene (Esi0080_0017) and the M1Pase gene (Esi0080_0016) in Ectocarpus. A full-length sequence of a SjM2DH gene and its high activity on DFructose reduction reaction has been reported [27]. Unexpected, the SjM2DH gene (SJ22304) was significantly down-regulated at the stage of SJsp5 compared to the other three stages (Figure 5), suggesting complex and unknown regulatory mechanism of this gene in reversible catalysis between mannitol and fructose. Seven hexokinase (HK) genes were found in S. japonica according to the KEGG annotation but no SjFrK gene was identified by local tblastx search using Esi0139_0025 as query sequence, which is regard as candidate FrK gene in Ectocarpus [14], suggesting that there may be no 
fructose-specific kinase to catalyze the fructose into fructose-6-phosphate in S. japonica and the enzymes function in a fructokinase role needs further identification.

\section{Analysis of putative genes related to laminarin and trehalose biosynthesis}

Laminarin is also a unique storage carbohydrate compound in brown algae. There are 11 genes may be involved in laminarin biosynthesis pathway in S. japonica, including two glucose-6-phosphate isomerase (GPI) genes, one phosphoglucomutase (PGM) gene, one UDP-glucose pyrophosphorylase (UGP) gene, one fusion PGM/UPG protein gene, three beta-1,3-glucan synthases (GS) genes and three beta-1,6-glucan active enzymes (GH16 family) genes (Additional file 6: Figure S9). The SjGPI gene SJ08179 displayed a very higher expression level than the SjGPI gene SJ00085 during the sporophyte development, and showed a significantly up-regulated expression in stage of SJsp3, SJsp5 and SJsp6, suggesting that the gene SjGPI-SJ08179 may play a key role in catalyzing the F6P to G6P. Except a single PGM gene (SJ12510) and a single UGP gene (SJ10502), there is a fusion PGM/UGP protein gene (SJ18341) was identified, which encoded a biofunctional enzyme. Although little UGP and PGM activities were detected in this fusion protein [24], the fusion protein SjPGM/UGP gene showed a constant higher expression levels than the single SjPGM gene and the SjUGP gene during sporophyte development in this study. Three members of GT48 family have been reported to be the putative GSs in Ectocarpus [15], and their orthologs from S. japonica were found, respectively. Among them, SjGS-SJ14967 and its orthologs from E. siliculosus (CBJ32439) clustered into a clade, which also including those from diatoms and Oomycetes, suggesting SJ14967 may be involved in the polymerization of the backbones of laminarin (Additional file 6: Figure S10). Expression analysis showed that the gene SJ07490 and SJ14967 have similar expression patterns, indicating that they might be functionally cooperative. The high expression level for SJ05913 was measured, suggesting its potential role in polymerization of other $\beta$-1, 3-glucans (e.g. callose). In addition, three members of GH16 were identified to be involved in $\beta$-1, 6 -branching of laminarin. The gene SJ07306 was persistently highly expressed, whereas the gene SJ17242 and SJ06967 showed preferential expression in the stage of SJsp5, indicating their different roles in laminarin modification during sporophyte development.

Trehalose is the most commonly occurring nonreducing disaccharides and the trehalose metabolism pathway has been constructed in Ectocarpus based on genome annotation data. Trehalose was formed from glucose-6-phosphate and UDP-glucose by trehalose-6-phosphate synthase (TPS) and trehalose-5phosphate phosphatase (TPP), and the recycling of trehalose is catalyzed by trehalase. Four TPS/TPP fusion protein genes were identified in S. japonica, except for a reported TPS/TPP gene (KC578568) [26]. Phylogenetic analysis showed that SjTPS/TPPs were divided into two groups (Additional file 6: Figure S11). One group consists of three SjTPS/TPPs and their orthologs from Ectocarpus and diatoms. In another group, two SjTPS/TPPs cluster with their orthologs from Ectocarpus and oomycetes into two subcaldes. The expression analysis showed that the four SjTPS/TPP genes had a consistent expression pattern. The expression levels of them increased in the stage of SJsp3 and SJsp5, while decreased in the stage of SJsp6, indicating that they might be cooperatively involved in trehalose synthesis. 
Verification of DEGs expression by qRT-PCR

To validate the genes expression levels during sporophyte development, ten unigenes related to cell wall polysaccharide and central storage carbon metabolism were selected for qRT-PCR analysis. The results showed that the relative transcript levels of the genes examined by qRT-PCR were largely consistent with those measured by RNA-Seq (Figure 6), which verified the precision and reliability of the transcriptome analysis results.

\section{Discussion}

Storage carbohydrates are various, and their biogenesis are very complicated in brown algae. The content of each carbohydrate is influenced not only by the type of species but also by its environmental conditions, its maturity and also the season [31]. In order to understand the dynamic changes of carbohydrate content and its correlation with the carbohydrate biosynthesis genes in the cultured kelp $S$. japonica, the sporophytes were sampled monthly and the contents of carbohydrates, represented by alginate and mannitol, were determined. In addition, the sporophyte samples used in this study were obtained by gametophyte cloning hybrid method, which can reduce the genetic background noise. The results showed that alginate and mannitol content significantly fluctuated among four stages (SJsp1, SJsp3, SJsp5 and SJsp6), indicating these four stages were critical for carbohydrate metabolism in $S$. japonica. Meanwhile, the phenotypes of thallus also showed distinct characteristics at these stages (Figure 1c). In the SJsp1 stage, the square concave and convex parts appear at the middle of the blade, which is approximately $1 \mathrm{~m}$ in length. In the SJsp3 stage, the blade is light brown, smooth, crisp with high water content and easy to break. The growth rate is highest in length and width. Subsequently, the growth rate in length and width decreased, while the thickness and dry weight increased faster in the SJsp5 stage, and the blade become dark brown and flexible. At the SJsp6 stage, sporangiums appear on the surface of blade. According to the above phenotypic characteristics of thallus and the biochemical analysis of alginate and mannitol content, the development of $S$. japonica sporophytes was divided into four different stages for subsequent transcriptome analysis.

More than $589 \mathrm{M}$ high-quality reads were generated by 12 transcriptomic libraries sequenced by the Illumina platform. Using these extensive RNA-Seq data, 4,514 novel genes and 27,079 novel transcripts for known genes were identified by annotation of different transcriptomes, which improves the functional annotation associated with the protein-coding genes and provides an important resource for insight into the specific biological process studies in S. japonica. Differential gene expression analysis suggested that a large number of genes were differentially expression and could be classified into different cluster with different expression patterns in four developmental stages. These genes with similar expression patterns might be functionally correlated during sporophytes development. Highest percentage of genes were group in "metabolic process", "cell" and "catalytic activity" in each of the three main categories by GO enrichment analysis, and the gene associated with "Carbon metabolism", "Photosynthesis", and "Photosynthesis-antenna proteins" pathways were significantly enriched. These results may allow for the identification of novel genes involved in the carbohydrates metabolite biosynthesis pathways from $S$. 
japonica. In addition, some lipid metabolism pathways were also enriched, indicating that the transcriptomic data in this study were also of potential value for identification of candidate genes and understanding molecular regulation mechanism underlying the lipid metabolism in S. japonica.

The components of the cell wall of brown algae mainly include alginate, fucoidan and cellulose. Among them, the content of alginate is the highest, which may contribute up to $40 \%$ of dry weight in Laminariales [32]. In this study, 31 genes covering all known enzymatic steps in the biosynthesis of Ectocarpus alginate were identified in $S$. japonica. For most enzymatic steps, more than one gene was found and they showed different expression patterns, indicating their different functions and roles. Combining with the biochemical analysis results, several candidate genes whose expression coincided with changes of alginate content, might play critical roles in regulating the formation of alginate biosynthesis. For example, the expression pattern of SjGMD3-SJ11033 was consistent with the changes of alginate content and confirmed by qRT-PCR analysis, suggesting that it should be the candidate key gene in alginate biosynthesis in S. japonica. In the SJsp5 stage, the expression level of this gene and the content of alginate were significantly reduced at the same time. It is likely that the photosynthetically assimilated carbons are more diverted into cytoplasm carbohydrates (e.g mannitol and laminarin) rather than cell wall carbohydrates at this stage. This is consistent of the sporophytic phenotype changes, including the decrease of growth rate, the increase of thickness and the enhancement of toughness, and is verified by the higher expression level of the genes involved in cytoplasm carbohydrate biosynthesis (Figure 5 and Additional file 6: Figure S9). In addition, the SjGMD gene SJ03911 might also be cooperatively involved in this step due to its similar expression pattern with SJ11033 and SJ11025, although it was not DEG in sporophytes development. As found in Ectocarpus, maximum number of genes was observed for encoding putative MC5Es in S. japonica suggested that this gene might have undergone various gene duplication events during evolution and was responsible for different physical properties of alginate. Among the nine SjMC5E genes with differentiate expression during sporophytes development, five genes homologous to MC5E genes found in L. digitata, and they exhibited the different expression patterns, indicating that these specialized genes were involved in alginate modification by catalyzing the epimerization of $\mathrm{M}$ to $\mathrm{G}$ in a temporal dependent manner for environment adaption. In this study, all known enzymatic step-related genes for fucoidan biosynthesis from GDP-mannose were also found in $S$. japonica. Meanwhile, a bifunctional enzyme coding gene (SjFK-SJ13948) was identified, which could catalyze L-fucoseinto GDP-fucose. These results confirmed that the two previously reported pathways involved in the fucoidan synthesis may be present in S. japonica and provided good candidates for future functional studies. Cellulose is a common constituent in the cell wall of eukaryotes. Cellulose synthase (CESA) is an essential catalyst for the generation of cell wall cellulose. Many CESA genes have been revealed to involve in cellulose biosynthesis and modification [33]. Among the SjCESA genes identified, one SjCESA gene (SJ01458) and one SjCSL gene (SJ21252) were identified to be differentially expressed during sporophyte development, with the preferential expression in SJsp3 compared to in SJsp1. Combined with the developmental characteristics, sporophytes in stage SJsp3 was in the rapid growth period, with the highest growth rate in length and width. This finding suggests that these two genes might 
play a key role in providing the carbon backbone necessary for cell growth, proliferation and development in S. japonica.

Mannitol and laminarin are interchangeable storage carbohydrates in brown algae, as are sucrose and starch in higher plants [3]. A complete set of enzymes for biosynthesis of mannitol and laminarin were found, but enzymes involved in the pathways for sucrose and starch were absent in S. japonica, which are consistent with previous studies in model brown alga Ectocarpus [14]. Mannitol is a unique storage carbohydrate in brown algae, which has not been reported in other Stramenopile, including diatoms and oomycetes. The standalone M1PDH genes and M1Pase genes were identified in S. japonica and were respectively clustered with their orthologs in Ectocarpus, suggesting that these genes were conserved in the evolution of brown algae. Of them, the SjM1DPH gene (SJ20113) and the SjM1Pase gene (SJ20112) were located next to each other on the genome and formed a functional cluster, which might be play a crucial role in mannitol biosynthesis. In addition, the phylogenetic analysis of M1PDH and M1Pase were consistent with previous study in Ectorcarpus, supporting the hypothesis that the mannitol-related genes in Micromonas were acquired recently from the brown alga by the horizontal gene transfer (HGT) [14]. The biochemical analysis of mannitol content showed that the accumulation pattern of mannitol is different from that of alginate in sporophyte development in S. japonica. Mannitol content did not decrease significantly in the SJsp5 stage compared to the SJsp3 stage. This result is confirmed by expression analysis of the candidate genes for mannitol biosynthesis (e.g SJ15139, SJ20113, SJ20112 and SJ07911), which maintained the high expression level in the SJsp5 stage (Figure 5). Moreover, the consistent expression patterns were also detected in candidate genes for laminarin biosynthesis (Additional file 6: Figure S9), providing evidences for the hypothesis that storage carbohydrates biosynthesis pathway in cytoplasm are predominantly activated in the SJsp5 stage. However, we noticed that the expression of most candidate genes for mannitol synthesis was down-regulated in the stage SJsp6 whereas the mannitol content increased significantly. It has been reported that mannitol can be remobilized and translocated from mature tissues to provide $\mathrm{C}$ for algae rapid growth [34]. There is also an interchangeable relationship between mannitol and laminarin during the carbohydrate metabolism process. Thus, the more complex regulatory mechanisms involved in mannitol biosynthesis, modification and conversion need to be further studied. Trehalose is found in a large range of bacteria and eukaryotes and is considered to be an ancient metabolite [35]. Three SjTPS/TPPs and their orthologs from Ectocarpus and diatoms form a group, which have been reported to be originated from red algae TPS/TPP (XP_005536433.1). Two SjTPS/TPPs along with their orthologs from Ectocarpus and oomycetes are derived from the red algae TPS/TPPs XP_005537475.1 and XP_005537876.1, respectively [14]. These results provide evidence that brown algae acquired trehalose metabolism by endosymbiosis of red algae.

\section{Conclusions}

In this study, we performed RNA-Seq-based transcriptome analysis of S. japonica sporophytes to identify genes involved in carbohydrates biosynthesis. The phenotypic characteristics and the dynamitic changes of alginate and mannitol content revealed that the sporophytes development can be divided into four 
different stages. Comparative transcriptome analysis among the four developmental stages led to the identification of candidate genes involved in the biosynthesis of carbohydrates including alginate, mannitol, fucoidan, cellulose, laminarin, and trehalose. The work presented is the first transcriptome analysis of sporophytes development and provides a dynamic view of carbohydrate metabolism process in $S$. japonica. Our results provide useful information to elucidate the molecular mechanism of carbohydrates metabolism and its regulation in S. japonica and may be used for breeding of carbohydrates-related traits in kelp to enhance commercial values.

\section{Methods}

\section{Biological material}

Kelp in Chinese aquaculture refers to a cultured species, S. japonica (Areschoug) CE Lane, C Mayes, LD Druehl \& GW Saunders (Phaeophyceae, Laminariales). In order to obtain the biological materials with the same genetic background in theory, the diploid sporophytes of $S$. japonica were cultivated by gametophyte cloning hybrid method as described previously [36]. The male gametophyte clone and the female gametophyte clone of $S$. japonica used in this study were maintained at Aquacultural Elite Variety Factory, Shandong Oriental Ocean Sci-tec Co., Ltd., Yantai, Shandong Province, China. The sporophytes were grown in Chudao Island Experimental Field of National Engineering and Technique Research and

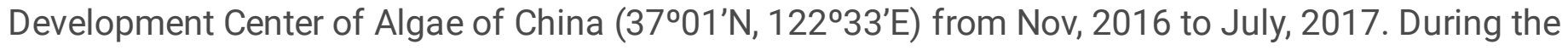
sporophytes developmental process, the materials were sampled monthly from Jan to July 2017, except June (SJsp1-SJsp6 stage, respectively). For phytochemical analysis, the nine whole blades of sporophytes with three biological replicates were sampled and were dried in each stage. For RNA-seq and qRT-PCR analysis, 9 individuals growing at the top layer of seawater were selected with three biological replicates. In each stage, a blade tissue discs with $2 \mathrm{~cm}$ in diameter was punched from the central band part of blade (50 $\mathrm{cm}$ to blade base), scrubbed with absorbent cotton, washed with sterile seawater, and stored at $-80^{\circ} \mathrm{C}$ after immediately frozen in liquid nitrogen.

\section{Determination of content of alginate and mannitol}

Alginate was extracted and measured according to Shang et al [37]. Briefly, $20 \mathrm{~g}$ of sun-dried alga was stirred for about $2 \mathrm{~h}$ in $500 \mathrm{~mL}$ of deionized water and $5 \mathrm{~g}$ of solid $\mathrm{Na}_{2} \mathrm{CO}_{3}$ at $80-90{ }^{\circ} \mathrm{C}$ until the algal sample were all paste-like. After dilution with deionized water and filtration, the alginate filtrate was acidification with $\mathrm{HCl}$ for $30 \mathrm{~min}(\mathrm{pH} 2.0)$ to form a gelatinous precipitate. Then, alginate precipitation was neutralized by $2 \mathrm{M} \mathrm{NaOH}$ to $\mathrm{pH} 7.0$, dehydrated by $95 \%$ ethanol, dried for $24 \mathrm{~h}$ at $45^{\circ} \mathrm{C}$ and milled before storage. Alginate yield was expressed as a percentage of dry weight (\% dw). The content of mannitol was determinate according to Cameron et al. and Zubia et al. and was also expressed as a percentage of dry weight $(\% \mathrm{dw})[38,39]$. 
Total RNA was extracted from individual tissues using the RNeasy Plant Mini Kit (Qiagen, Germany) according to manufacturer's instructions. RNA purity and RNA concentration were measured using Nanodrop 1000 spectrophotometer (ThermoFisher Scientific). RNA integrity was assessed using the Agilent 2100 Bioanalyzer (Agilent Technologies).

Twelve RNA sequencing libraries with three independent biological replicated analysis in four stages (SJsp1, SJsp3, SJsp5 and SJsp6) were prepared using IlluminaTruSeq RNA Sample Preparation Kit according to the manufacturer's instructions. Briefly, mRNA was purified from total RNA using oligo-(T) magnetic beads. Then, the mRNA was fragmented and transcribed into first strand cDNA using random hexamer-primers and SuperScript II. This was followed by second strand cDNA synthesis using DNA polymerase I and RNase $\mathrm{H}$. Then, the high-throughput RNA-sequencing libraries were prepared following Illumina's protocols and were sequenced on the Illumina Hiseq 4000 platform with 150 bp paired-end reads.

\section{Mapping of reads to the reference genome and predicting novel transcript}

Raw reads were filtered to obtain high-quality clean reads by removing reads with adaptors, reads in which unknown base $(\mathrm{N})$ are more than $5 \%$ and low quality reads (the percentage of base which quality is lesser than 15 is greater than $20 \%$ in a read) using software SOAPnuke ( $v$ 1.5.2; https://github.com/BGI-flexlab/SOAPnuke). After reads filtering, clean reads were mapped to the $S$. japonica reference genome (Genebank number: JXRI00000000.1) using the mapping software HISAT ( $v$ 2.0.4) with standard parameters (--phred64 -sensitive -no-discordant -no-mixed -I 1 -X 1000) [29].

The transcripts in this study were reconstructed using StringTie and were compared to reference annotation using Cuffcompare [40, 41]. After that, ' $\mathrm{u}$ ', ' I', ' $\mathrm{o}$ ', ' $\mathrm{j}$ ' class code types were selected as novel transcripts. Coding potential of novel transcripts were predicted using CPC, and then coding novel transcripts were merged with reference transcripts to get a complete reference [42]. All downstream analysis was conducted base on this reference.

\section{Quantification of gene expression levels}

For gene expression analysis, clean reads were mapped to reference using Bowtie2 and then readcount of each gene was estimated using RSEM in each library $[43,44]$. The FPKM value was used to assess the expression levels of unigenes.

\section{Identification of DEGs and clustering analysis}

Different expression genes (DEGs) among different samples were detected using DEG seq2 [45]. The absolute fold change $>2$ and adjusted $p$-value $<0.05$ were set as the threshold to judge the significant DEGs. The clustering analysis was performed using $\mathrm{R}$ package pheatmap [46]. 
On the basis of GO (Gene Ontology)/KEGG (Kyoto Encyclopedia of Genes and Genomes) annotation results, the $\mathrm{GO}$ categories and KEGG pathway enrichment analysis of DEGs was performed using $R$ package phyper. The false discovery rate (FDR) was calculated for each $p$-value, and the terms with FDR $\leq 0.01$ were defined as significant enriched.

Analysis of genes involved in cell wall polysaccharide and central storage carbon metabolism

According to the KEGG annotation and local blast search with a cutoff E value of $1 e-5$, the genes involved in cell wall polysaccharide metabolism and central storage carbon metabolism, especially the biosynthesis of alginate and mannitol, were mined and analyzed. The query sequences used for local blast search were the corresponding homologous gene functionally characterized in other brown algal species. To distinguish the orthologs of corresponding functionally characterized genes within larger-size gene family, phylogenetic and molecular evolutionary analysis were done using the neighbor-joining method with MEGA6 after the amino acid sequences were aligned using ClustalW [47]. The proteins used for phylogenetic analysis were listed in Additional file 4: Table S4.

\section{Real-time quantitative PCR analysis of DEGs}

RNA extraction was performed as described above. cDNA was synthesized using the PrimeScript RT Reagent Kit with gDNA Eraser (TaKaRa). Ten genes were selected for validation using quantitative realtime PCR. The EF1 a gene was used as the reference for internal standardization. All the primers were designed using Primer3 and are listed in Additional file 5: Table S5 [48, 49]. The qRCR reaction was performed on a CFX96 real-time PCR Detection System (Bio-Rad Laboratories, CA) following the manufacturer's protocol for SYBR Premix Ex Taq ${ }^{\mathrm{TM}}$ (TaKaRa). The PCR conditions were as follows: an initial step at $95^{\circ} \mathrm{C}$ for $30 \mathrm{~s}$; followed by 40 cycles of denaturation at $95^{\circ} \mathrm{C}$ for $5 \mathrm{~s}$ and annealing at $59^{\circ} \mathrm{C}$ for $5 \mathrm{~s}$; and a final dissociation curve analysis, $5 \mathrm{~s}$ at $65^{\circ} \mathrm{C}$, then up to $95^{\circ} \mathrm{C}$ at a rate of $0.5^{\circ} \mathrm{C} / \mathrm{s}$. The data were exported and quantified via the comparative $\mathrm{Ct}$ method $\left(2^{-\Delta \Delta C t}\right)$ [50]. All the experiments were repeated using three biological replicates the data were analyzed statistically ( \pm Standard Deviation).

\section{Abbreviations}

GMD: GDP-mannose-6-dehydrogenase; MC5E: mannuronate C5-epimerase; MPI : mannose-6-phosphate isomeras; PMM: phosphomannomutase; MPG: mannose-1-phosphate guanylyltransferase; PGM: phosphoglucomutase; MS: mannuronan synthase; GM46D: GDP-mannose 4, 6-dehydratase; GFS: GDPfucose synthetase; FS: fucosyltransferase; ST: sulfotransferase; FK: fucokinase; GFPP: GDP-fucose pyrophosphorylas; CESA: cellulose synthase; CSL: cellulose synthase-like; M1PDH: mannitol-1-phosphate5-dehydrogenase; M1Pase: mannitol-1-phosphatase; M2DH: mannitol-2-dehydrogenase; FrK: fructokinase; HK: hexokinase; GPI: glucose-6-phosphate isomerase; UGP: UDP-glucose pyrophosphorylase; GS: beta-1,3-glucan synthases; TPS: trehalose-6-phosphate synthase; TPP: trehalose5-phosphate phosphatase; HGT: horizontal gene transfer; DEGs: differentially expressed genes; SRA: 
Sequence Read Archive; GO: Gene Ontology; KEGG: Kyoto Encyclopedia of Genes and Genomes; FDR: false discovery rate.

\section{Declarations}

Ethics approval and consent to participate

Not applicable.

Consent for publication

Not applicable.

Availability of data and material

All raw RNA sequence read data have been deposited in NCBI Sequence Read Archive (SRA) under accession PRJNA511944.

Competing interests

The authors declare that they have no competing interests.

Acknowledgements

The authors greatly appreciate the assistances of Zhuangzhi Zhang, Shandong Oriental Ocean Sci-tech Co., Ltd, Yantai, Chian, in seedling raising and sporophyte field culturing.

Funding

This study was financially supported by the National Natural Sciences Foundation of China (Grant no. 41606184), "First Class Fishery Discipline" Programme in Shandong Province China, Agricultural Scientific and Technological Innovation Project of Shandong Academy of Agricultural Sciences (Grant no. CXGC2016B10) and the Qingdao Agricultural University Foundation (Grant no. 663-1118010).

Authors' contributions

Study conception and design: LNZ, XJL, HW. Physicochemical analysis: CZM, HW. Sample collection: GJL, XJL. RNA extraction, analysis and interpretation of RNA-seq data: LNZ, HW. Manuscript: LNZ, HW. All authors reviewed and approved the final version of the manuscript.

\section{Reference}

1. Baldauf SL. An overview of the phylogeny and diversity of eukaryotes. J Syst Evol. 2008;46:263-73. 
2. Kloareg B, Mabeau S. Isolation and analysis of the cell walls of brown algae: Fucus spiralis, $F$. ceranoides, F. vesiculosus, F. serratus, Bifurcaria bifurcata and Laminaria digitata. J Exp Bot. 1987;38:1573-80.

3. Yamaguchi T, Ikawa T, Nisizawa K. Incorporation of radioactive carbon from $\mathrm{H}^{14} \mathrm{CO}_{3}{ }^{-}$into sugar constituents by a brown alga, Eisenia bicyclis, during photosynthesis and its fate in the dark. Plant Cell Physiol. 1966;7:217-29.

4. Daroch M, Geng S, Wang G. Recent advances in liquid biofuel production from algal feedstocks. Appl Energy. 2013;102:1371-81.

5. Lee KY, Mooney DJ. Alginate: Properties and biomedical application. Prog Polym Sci. 2012;37:106-26.

6. Pawar SN, Edgar KJ. Algiante derivatization: a review of chemistry, properties and applications. Biomaterials. 2012;33:3279-305.

7. Wei N, Quarterman J, Jin YS. Marine macroalgae: an untapped resource for producing fuels and chemicals. Trends Biotechnol. 2013;31:70-7.

8. Enquist-Newman M, Faust AM, Bravo DD, Santos CN, Raisner RM, Hanel A, et al. Efficient ethanol production from brown macroalgae sugars by a synthetic yeast platform. Nature. 2014;505:239-43.

9. Wargacki AJ, Leonard E, Win NM, Regitsky DD, Santos CN, Kim PB, et al. An engineered microbial platform for direct biofuel production from brown macroalgae. Science. 2012;335:308-13.

10. Xia A, Jacob A, Herrmann C, Tabassum MR, Murphy JD. Production of hydrogen, ethanol and volatile fatty acids from the seaweed carbohydrate mannitol. Bioresour Technol. 2015;193:488-97.

11. Cock JM, Sterck L, RouzéP, Scornet D, Allen AE, Amoutzias G, et al. The Ectocarpus genome and the independent evolution of multicellularity in brown algae. Nature. 2010;465:617-21.

12. Bonin P, Groisillier A, Raimbault A, Guibert A, Boyen C, Tonon T. Molecular and biochemical characterization of mannitol-1-phsphate dehydrogenase from the model brown alga Ectocarpus sp. Phytochemistry. 2015;117:509-20.

13. Groisillier A, Shao Z, Michel G, Goulitquer S, Bonin P, Krahulec S, et al. Mannitol metabolism in brown algae involves a new phosphatase family. J Exp Bot. 2014;65:559-70.

14. Michel G, Tonon T, Scornet D, Cock JM, Kloareg B. Central and storage carbon metabolism of the brown alga Ectocarpus siliculosus: insights into the origin and evolution of storage carbohydrates in Eukaryotes. New Phytol. 2010a;188:67-81.

15. Michel G, Tonon T, Scornet D, Cock JM, Kloareg B. The cell wall polysaccharide metabolism of the brown alga Ectocarpus siliculosus: insights into the evolution of extracellular matrix polysaccharides in 
Eukaryotes. New Phytol. 2010b;188:82-97.

16. Rousvoal S, Groisillier A, Dittami SM, Michel G, Boyen C, Tonon T. Mannitol-1-phosphate dehydrogenase activity in Ectocarpus siliculosus, a key role for mannitol synthesis in brown algae. Planta. 2011;233:261-73.

17. Tenhaken R, Vogals E, Cock JM, Neu V, Huber CG. Characterization of GDP-mannose dehydrogenase from the brown alga Ectocarpus siliculosus providing the precursor for the alginate polymer. J Biol Chem. 2011;286:16707-15.

18. Adams JM, Ross AB, Anastaskis K, Hodgson EM, Gallagher JA, Jones JM, et al. Seasonal variation in the chemical composition of the bioenergy feedstock Laminaria digitata for thermochemical conversion. Bioresour Technol. 2011;102:226-34.

19. Rosell KG, Srivastava LM. Seasonal variation in the chemical constituents of the brown algae Macrocystis integrifolia and Nereocystis luetkeana. Can J Bot. 1984; 62:2229-36.

20. Schiener P, Black KD, Stanley MS, Green DH. The seasonal variation in the chemical composition of the kelp species Laminaria digitata, Laminaria hyperborea, Saccharina latissima and Alaria esculenta. J Appl Phycol. 2015;27:263-73.

21. Zimmerman RC, Kremer JN. In situ growth and chemical composition of the giant kelp, Macrocystis pyrifera: response to temporal changes in ambient nutrient availability. Mar Ecol Prog Ser. 1986;27:27785.

22. Sheng XF, Zhao YF, Shang DR, Ning JS, Guo YY. Differences in the major nutrition constituents and major elements in the kelp of different growing stages. Food Sci Technol. 2011;36:66-68 (in Chinese with English Abstract).

23. Ye N, Zhang X, Miao M, Fan X, Zheng Y, Xu D, et al. Saccharina genomes provide novel insight into kelp biology. Nat Commun. 2015;6:6986.

24. Chi S, Feng YJ, Liu T. Molecular cloning, characterization, and comparison of UDP-glucose pyrophosphorylase from Gracilaria chouae and Sacharina japonica. J Appl Phycol. 2016;28:2051-9.

25. Chi S, Liu T, Wang X, Wang R, Wang S, Wang G, et al. Functional genomics analysis reveals the biosynthesis pathways of important cellular components (alginate and fucoidan) of Saccharina. Curr Genet. 2018;64:259-73.

26. Deng Y, Wang X, Guo H, Duan D. A trehalose-6-phosphate synthase gene from Saccharina japonica (Laminariales, Phaeophyceae). Mol Biol Rep. 2014;41:529-36.

27. Shao Z, Zhang P, Li Q, Wang X, Duan D. Characterization of mannitol-2-dehydrogenasein Saccharina japonica: evidence for a new polyol-specific long-chain dehydrogenases/reductase. PLoS ONE. 2014;9: 
e97935.

28. Zhang PY, Shao ZR, Li L, Liu S, Yao JT, Duan DL. Molecular characterisation and biochemical properties of phosphomannomutase/phosphoglucomutase (PMM/PGM) in the brown seaweed Saccharina japonica. J Appl Phycol. 2018;30:2687-96.

29. Kim D, Langmead B, Salzberg SL. HISAT: a fast spliced aligner with low memory requirements. Nat Methods. 2015;12:357-60.

30. Hay ID, Ur Rehamn Z, Ghafoor A, Rehm BHA. Bacterial biosynthesis of alginates. J Chem Technol Biot. 2010;85:752-9.

31. Murakami K, Yamaguchi Y, Noda K, Fujii T, Shinohara N, Ushirokawa T, et al. Seasonal variation in the chemical composition of a marine brown alga, Sargassum horner(Turner) C. Agardh. J Food Compos Anal. 2011;24:231-6.

32. Horn SJ, Moen E, Østgaard K. Direct determination of alginate content in brown algae by near infrared (NIR) spectroscopy. J Appl Phycol. 1999;11:9-13.

33. Liepan AH, Wightman R, Greshi N, Turner SR, Scheller HV. Arabidopsis - a powerful model system for plant cell wall research. Plant J. 2010;61:1107-21.

34. Lobban CS, Harrison PJ. Seaweed ecology and physilolgy, 2nd ed. Cambridge University Press; 1994.

35. Goddijn O J, van Dun K. Trehalose metabolism in plants. Trends Plant Sci. 1999;4:315-9.

36. Li XJ, Cong YZ, Yang GP, Shi YY, Qu SC Li ZL, et al. Trait evaluation and trial cultivation of Dongfang No. 2, the hybrid of a male gametophyte clone of Laminaria longissima (Laminariales, Phaeophyta) and a female one of L. japonica. J Appl Phycol. 2007;19:139-51.

37. Shang DR, Ning JS, Zhao YF, Zhai YX, Shu BS, Sheng XF, et al. Establishment of the determination on kelp alginate. Food Sci Technol. 2011;36:252-4 (in Chinese with English Abstract).

38. Cameron MC, Ross AG, Percival EGV. Methods for the routine estimation of mannitol, alginic acid and combined fucose in seaweed. J Soc Chem Ind. 1948;67:161-4.

39. Zubia M, Payri C, Deslandes E. Alginate, mannitol, phenolic compounds and biological activities of two range-extending brown algae, Sargassum mangarevense and Turbinaria ornata (Phaeophyta: Fucales), from Tahiti (French Polynesia). J Appl Phycol. 2008;20:1033-43.

40. Pertea M, Pertea GM, Antonescu CM, Chang TC, Mendell JT, Salzberg SL. StringTie enables improved reconstruction of a transcriptome from RNA-seq reads. Nat Biotechnol. 2015;33:290-5.

41. Trapnell C, Roberts A, Goff L, Pertea G, Kim D, Kelley DR, et al. Differential gene and transcript expression analysis of RNA-seq experiments with TopHat and Cufflinks. Nat Protoc. 2012;7:562-78. 
42. Kong L, Zhang Y, Ye ZQ, Liu XQ, Zhao SQ, Wei LP, et al. CPC: assess the protein-coding potential of transcripts using sequence features and support vector machine. Nucleic Acids Res. 2007;35:W345-9.

43. Langmead B, Salzberg S. Fast gapped-read alignment with Bowtie 2. Nat Methods. 2012;9:357-9.

44. Li B, Dewey CN. RSEM: accurate transcript quantification from RNA-Seq data with or without a reference genome. BMC Bioinformatics. 2011;12:323.

45. Love Ml, Huber W, Anders S. Moderated estimation of fold change and dispersion for RNA-seq data with DESeq2. Genome Biol. 2014;15:1-21.

46. Wang L, Feng Z, Wang X, Zhang X. DEGseq: an R package for identifying differentially expressed genes from RNA-seq data. Bioinformatics. 2010;26:136-8.

47. Tamura K, Stecher G, Peterson D, Filipski A, Kumar S. MEGA6: Molecular evolutionalry genetics analysis version 6.0. Mol Biol Evol. 2013;30:2725-9.

48. Koressaar T, Remm M. Enhancements and modifications of primer design program Primer3. Bioinformatics. 2007;23:1289-91.

49. Untergrasser A, Cutcutache I, Koressaar T, Ye J, Faircloth BC, Remm M, et al. Primer3 - new capabilities and interfaces. Nucleic Acids Res. 2012;40:e115.

50. Kenneth JL, Thomas DS. Analysis of relative gene expression data using real-time quantitative PCR and the 2- $\Delta \Delta$ Ct method. Methods. 2001;25:402-8.

\section{Additional File Legends}

Additional file 1: Table S1 Genes and Transcripts number based on RNA-seq data in four development stages of $S$. japonica sporophyte.

Additional file 2: Table S2 Differentially expressed genes among the different developmental stages.

Additional file 3: Table S3 Differentially expressed genes involved in KEGG pathway among the different develpmental stages.

Additional file 4: Table S4 List of sequences used for phylogenetic analyses and their corresponding GenBank accession number.

Additional file 5: Table S5 List of qRT-PCR primers used in this study.

Additional file 6: Figure S1 GO classification of DEGs. X axis represents number of DEG. Y axis represents GO term. Figure S2 Phylogenetic relationship of S. japonica GMD with those proteins of other species. The phylogenetic tree was constructed using the neighbor-joining method in MEGA 6. The bootstrap values are indicated by numbers at nodes. Dark solid triangle indicates the characterized SjGMD proteins 
in this study. Figure S3 Phylogenetic relationship of S. japonica PMM/PGM with those proteins of other species. The phylogenetic tree was constructed using the neighbor-joining method in MEGA 6 . The bootstrap values are indicated by numbers at nodes. Dark solid triangle indicates the characterized SjPMM/PGM proteins in this study. Figure S4 Phylogenetic relationship of S. japonica MC5E with those proteins of other species. The phylogenetic tree was constructed using the neighbor-joining method in MEGA 6. The bootstrap values are indicated by numbers at nodes. Dark solid triangle indicates the characterized SjMC5E proteins in this study. Figure S5 Expression patterns of genes encoding enzymes possibly involved in the fucoidan pathway in S. japonica. Fig S6 a: Phylogenetic analysis of cellulose synthase (CESA) and cellulose synthase-like (CSL). b: Expression patterns of SjCESA genes amd SjCSL genes. Asterisks represent differentially expressed genes. Figure $S 7$ Phylogenetic relationship of $S$. japonica M1PDH with those proteins of other species. The phylogenetic tree was constructed using the neighbor-joining method in MEGA 6. The bootstrap values are indicated by numbers at nodes. Dark solid triangle indicates the characterized SjM1PDH proteins in this study. Figure S8 Phylogenetic relationship of $S$. japonica M1Pase with those proteins of other species. The phylogenetic tree was constructed using the neighbor-joining method in MEGA 6. The bootstrap values are indicated by numbers at nodes. Dark solid triangle indicates the characterized SjM1Pase proteins in this study. Figure S9 Expression patterns

of genes encoding enzymes possibly involved in the laminarin pathway in $S$. japonica. Asterisks represent differentially expressed genes. Figure S10 Phylogenetic relationship of S. japonica GS with those proteins of other species. The phylogenetic tree was constructed using the neighbor-joining method in MEGA 6. The bootstrap values are indicated by numbers at nodes. Dark solid triangle indicates the characterized SjGS proteins in this study. Figure S11 Phylogenetic relationship of $S$. japonicaTPS/TPP with those proteins of other species. The phylogenetic tree was constructed using the neighbor-joining method in MEGA 6. The bootstrap values are indicated by numbers at nodes. Dark solid triangle indicates the characterized SjTPS/TPP proteins in this study.

\section{Tables}

Due to technical restrictions the tables for this paper have been attached as excel files in the supplementary files.

\section{Figures}



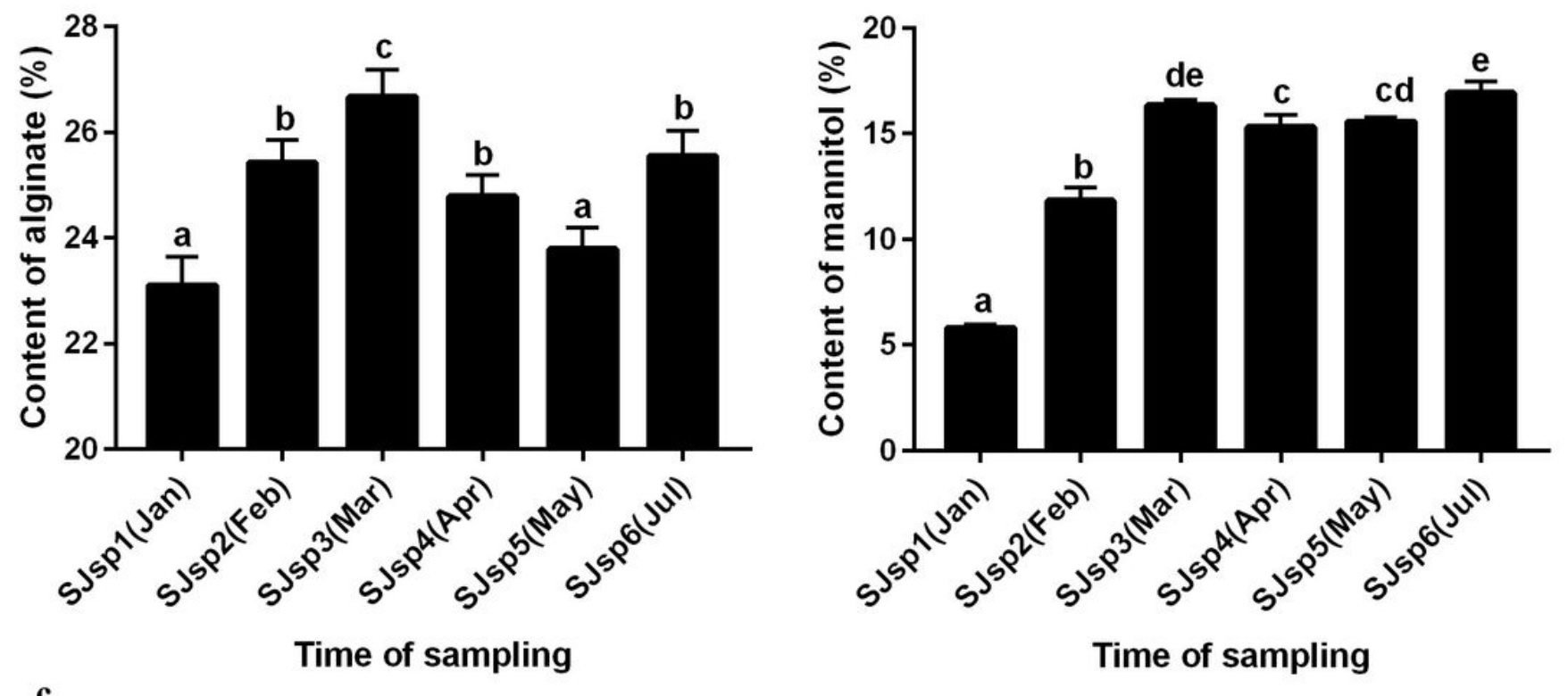

c
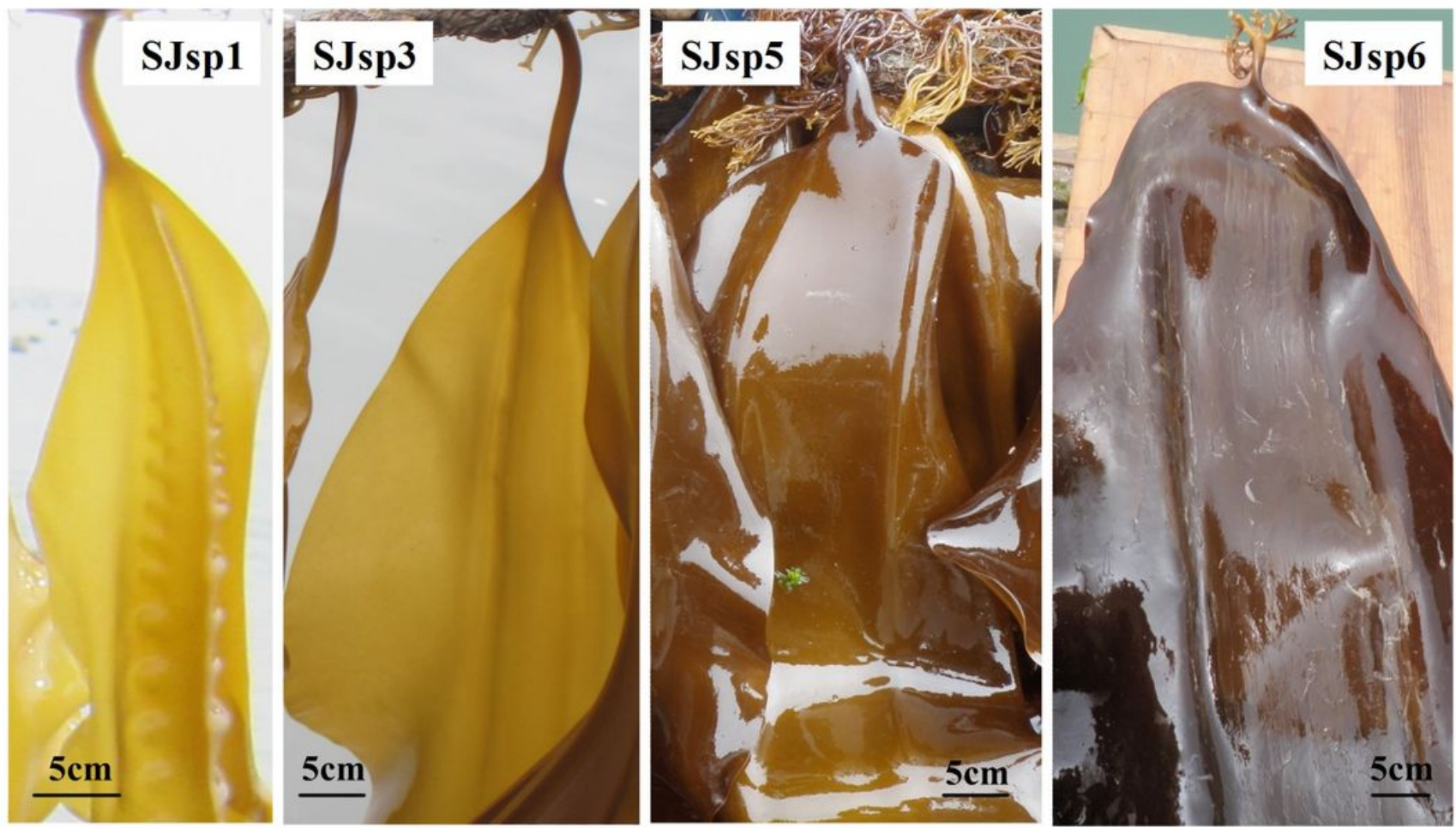

Figure 1

Changes of the alginate and mannitol content and phenotype during sporophyte development in S. japonica. A: Change of alginate content. Data are means $\pm S D(n=3) ; B$ : Change of mannitol content. Data are means $\pm S D(n=3)$. The same letters indicate no significant difference $(p>0.05)$; C: Photographs of sporophyte in SJsp1, SJsp3, SJsp5 and SJsp6, respectively. 


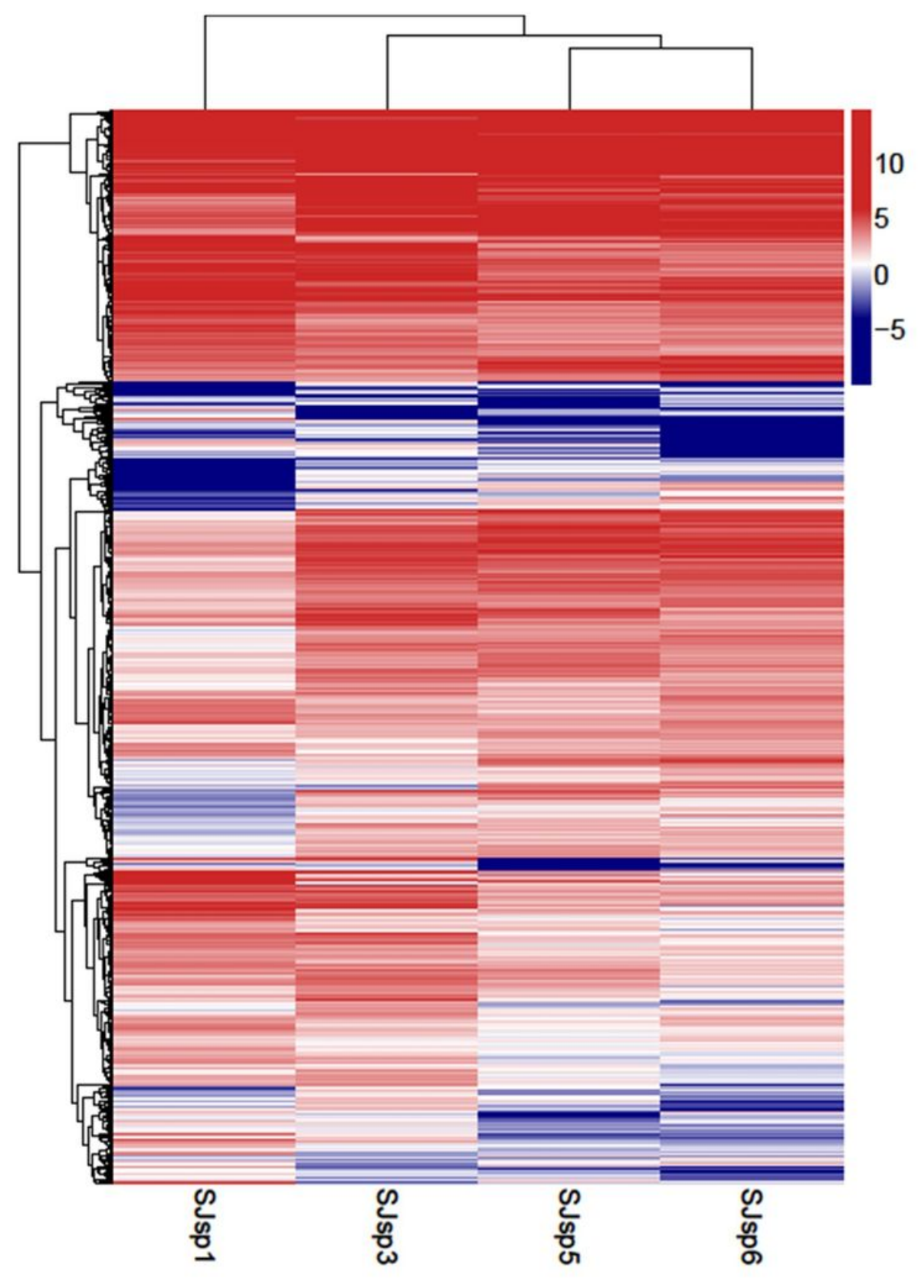

Figure 2

Cluster analysis of gene expression base on log10 FPKM value. Each column represents an developmental stage and each row represents a gene. Blue indicates lower expression and red indicates high expression. 


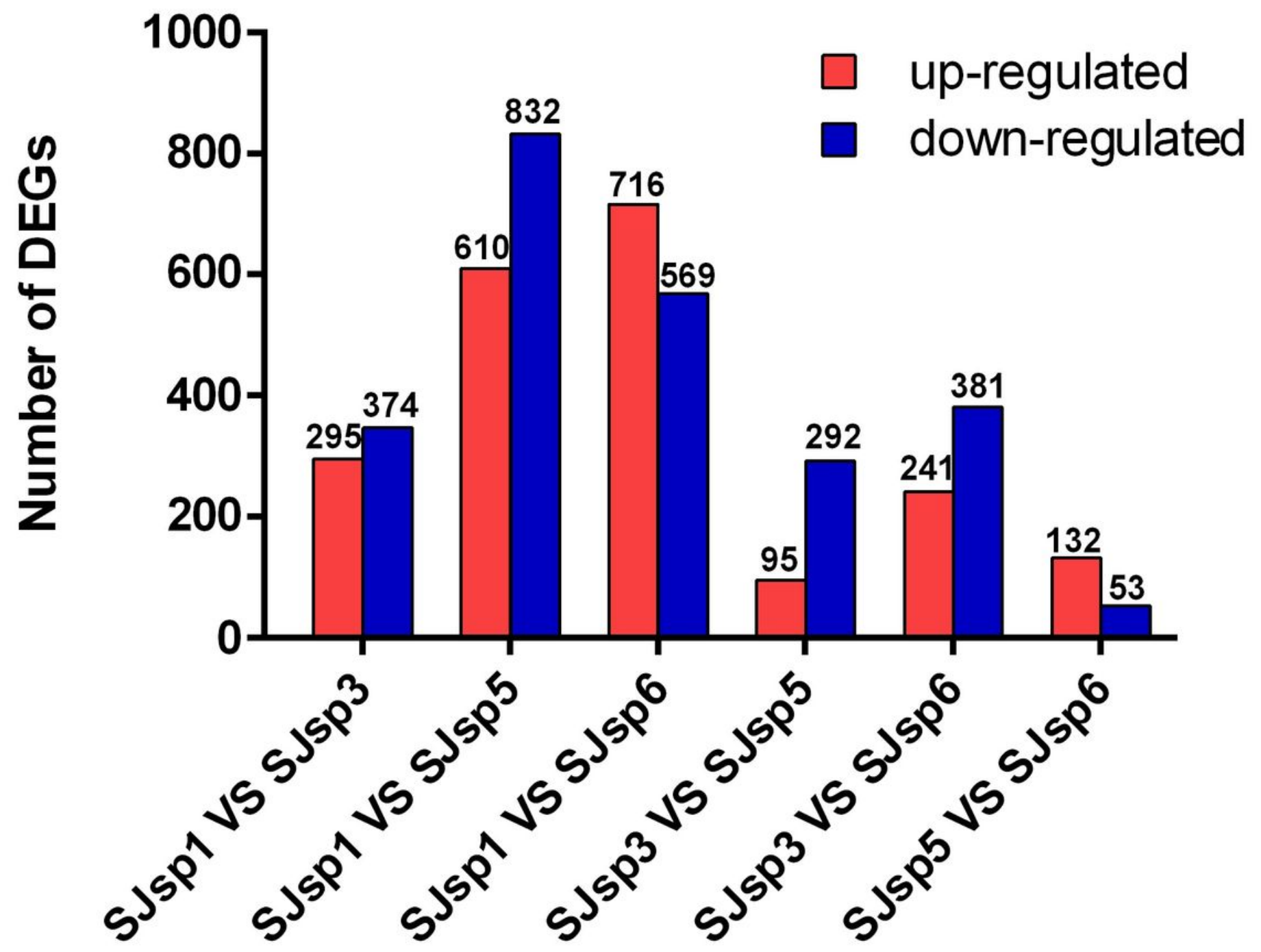

Figure 3

Different expression patterns of DEGs in different developmental stages. Red color represents upregulated DEGs. Blue color represents down-regulated DEGs. 


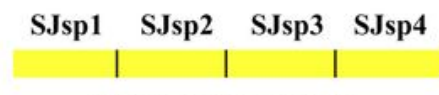

Developmental stage

Fructose-6-phosphate

MPI

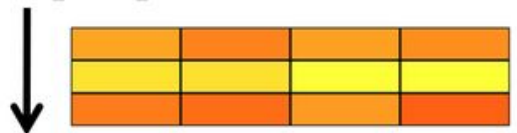

SjMPI1-SJ22044

SjMPI3-SJ15069

*SjMPI4-SJ15067

Mannose-6-phosphate

PMM

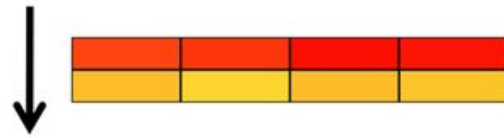

SjPMM-SJ14545

SjPMM/PGM-SJ02279

Mannose-1-phosphate
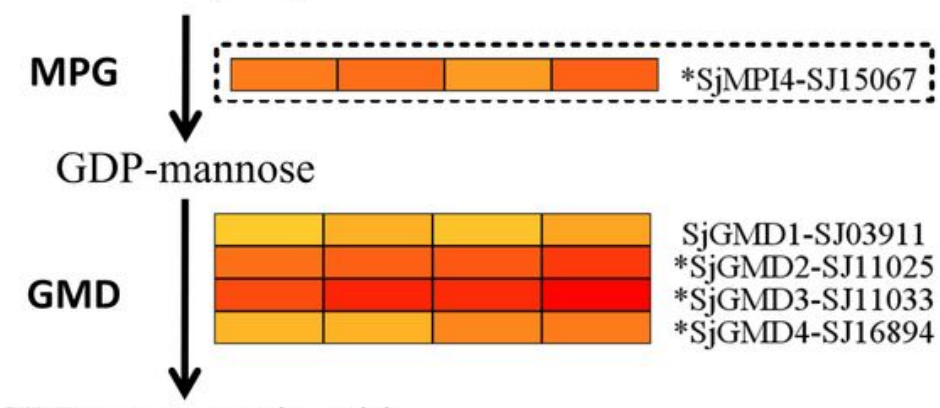

GDP-mannuronic acid

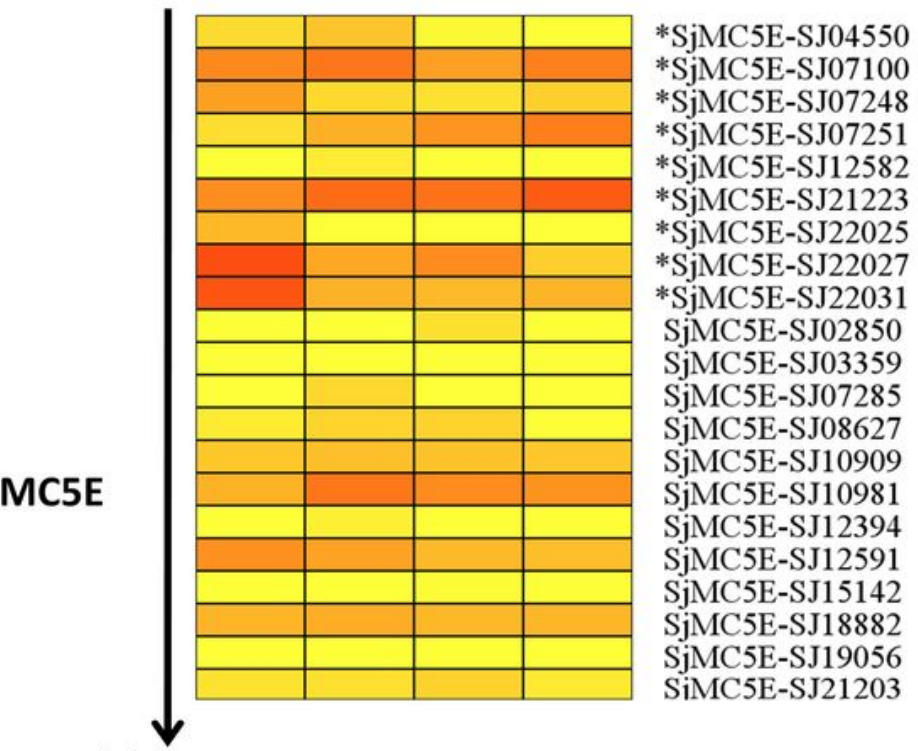

Alginate

0.0

$\log _{10}$ FPKM

3.3

\section{Figure 4}

Expression patterns of genes encoding enzymes possibly involved in the alginate pathway in S. japonica. Asterisks represent differentially expressed genes. 


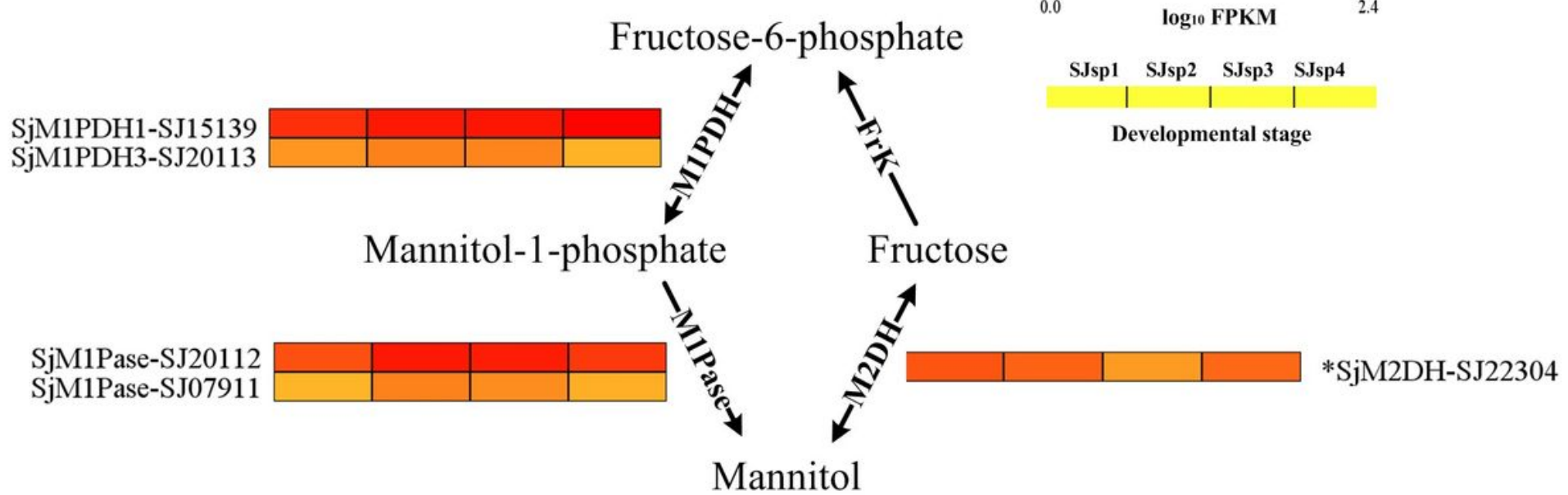

Figure 5

Expression patterns of genes encoding enzymes possibly involved in the mannitol pathway in S. japonica. Asterisks represent differentially expressed genes. 

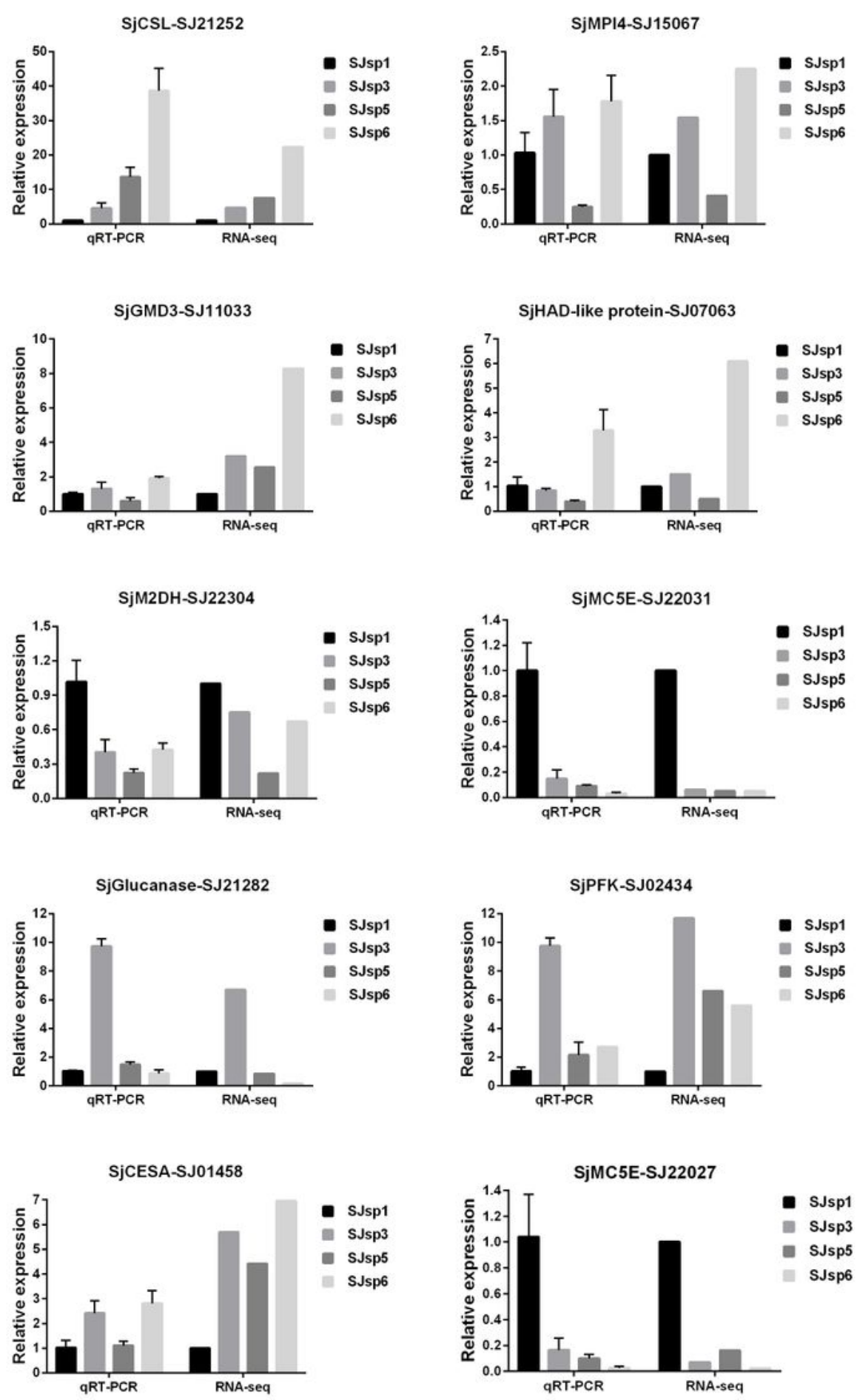

Figure 6

Validation of gene expression by qRT-PCR. The expression of the SJsp1 was set as 1.0, and the relative expression level was calculated. Error bars indicate standard deviation from three replicates of qRT-PCR. (1) Gene ID: SJ21252, Gene Name: Cellulose synthase; (2) Gene ID: SJ15067, Gene Name: phosphomannose isomerase 3; (3) Gene ID: 11033, Gene Name: UDP-glucose 6-dehydrogenase; (4) Gene ID: SJ07063, Gene Name: HAD-like protein; (5) Gene ID: SJ22304, Gene Name: mannitol dehydrogenase; 
(6) Gene ID: SJ22031, Gene Name: mannuronan C-5 epimerase; (7) Gene ID: SJ21282, Gene Name: Endo1,3-beta-glucanase; (8) Gene ID: SJ02434, Gene Name: pyrophosphate-dependent phosphofructose kinase; (9) Gene ID: SJ01458,Gene Name: cellulose synthase; (10) Gene ID: SJ22027, Gene Name: mannuronan C-5-epimerase.

\section{Supplementary Files}

This is a list of supplementary files associated with this preprint. Click to download.

- supplement1.xIsx

- supplement2.xlsx

- supplement3.xIsx

- supplement4.tif

- supplement5.tif

- supplement6.tif

- supplement7.tif

- supplement8.xlsx

- supplement9.xIsx

- supplement10.tif

- supplement11.xlsx

- supplement12.tif

- supplement13.xlsx

- supplement14.xlsx

- supplement15.tif

- supplement16.tif

- supplement17.tif

- supplement18.tif

- supplement19.tif 